Cahiers de Narratologie

Analyse et théorie narratives

13 | 2006

Nouvelles approches de l'intertextualité

\title{
Intertextualité et théorie
}

\section{Frank Wagner}

\section{OpenEdition}

Journals

Édition électronique

URL : http://journals.openedition.org/narratologie/364

DOI : 10.4000/narratologie.364

ISSN : 1765-307X

Éditeur

LIRCES

\section{Référence électronique}

Frank Wagner, «Intertextualité et théorie », Cahiers de Narratologie [En ligne], 13| 2006, mis en ligne le 01 septembre 2006, consulté le 19 avril 2019. URL : http://journals.openedition.org/narratologie/364 ; DOI : 10.4000/narratologie.364

Ce document a été généré automatiquement le 19 avril 2019

\section{(c) (i) $\odot$}

Cahiers de Narratologie - Analyse et théorie narratives est mis à disposition selon les termes de la licence Creative Commons Attribution - Pas d'Utilisation Commerciale - Pas de Modification 4.0 International. 


\title{
Intertextualité et théorie
}

\author{
Frank Wagner
}

1 La notion d'intertextualité, introduite par Julia Kristeva ${ }^{1}$, précisée notamment par Antoine Compagnon ${ }^{2}$ et Michael Riffaterre ${ }^{3}$, est redéfinie par Gérard Genette comme « une relation de coprésence entre deux ou plusieurs textes, c'est-à-dire, eidétiquement et le plus souvent, [...] la présence effective d'un texte dans un autre $»^{4}$, présence plus ou moins explicite qui recouvre les pratiques de la citation, du plagiat et de l'allusion.

2 Cette dernière, qui consiste en la production « d'un énoncé dont la pleine intelligence suppose la perception d'un rapport entre lui et un autre auquel renvoie nécessairement telle ou telle de ses inflexions, autrement non recevable $»^{5}$, implique par essence, en raison de sa dimension implicite, un travail d'induction de la part des lecteurs. Or, il me semble que face à nombre de textes, particulièrement contemporains, cette participation lectorale active ne se borne pas aux opérations de repérage puis décryptage des allusions intertextuelles, mais présente en outre une dimension proprement théorique. En effet, dans la mesure où tout texte littéraire exemplifie à quelque degré (évidemment et éminemment variable) la conception ou plutôt, n'ayons pas peur des mots, la théorie de la littérature qui l'informe, les opérations intertextuelles en constituent un mode d'expression privilégié. En grande partie parce qu'intertexte et métatexte ont toujours plus ou moins partie liée ${ }^{6}$ : la relation d'interlocution est aussi, inévitablement, relation de commentaire ${ }^{7}$.

3 Je souhaiterais mettre au jour ce poids théorique des opérations intertextuelles dans le roman de Raymond Queneau intitulé Le Vol d'Icare ${ }^{8}$. Sans doute est-il nécessaire de présenter sommairement ce texte que son foisonnement expérimental désigne tout naturellement à l'attention des théoriciens de la littérature. On peut le caractériser, au risque d'un léger anachronisme, comme une variation sur la notion de métalepse narrative, puisque l'essentiel de l'intrigue consiste dans les évasions (passage d'un niveau narratif supérieur à un autre, inférieur) des personnages créés par un quatuor d'écrivains imaginaires, leur circulation entre divers univers fictifs collatéraux, et leur venue finale à résipiscence. D'où une dialectique de l'autonomie et de l'arbitraire narratif, de la réalité 
et de la fiction, objets de récurrentes séquences métanarratives, partiellement déceptives car contradictoires.

4 A cela s'ajoute une spectaculaire (et en partie provocatrice) ambiguïté générique, puisque l'intitulé « roman » est maintenu pour désigner un texte constitué presque intégralement de séquences dialoguées au discours direct (présentées selon les normes typographiques du théâtre imprimé), liées par une rare parole narratoriale au discours indirect, d'apparence didascalique. De nouveau, cette tension entre modes narratif et dramatique fait l'objet de plusieurs commentaires métatextuels, délégués à divers personnages.

Mais c'est surtout la densité et la diversité intertextuelles de l'ouvrage qui attirent l'attention : parodie des milieux littéraires fin de siècle et allusions ponctuelles à l'œuvre de Flaubert ${ }^{9}$; choix du patronyme du protagoniste (Icare), objet d'un réseau d'allusions intertextuelles inauguré dès le paratexte par l'épigraphe empruntée à Ovide («Icare, dixit, ubi es? Qua te regione requiram?») et origine du télescopage ironique des mythologies ancienne et moderne ; et enfin intertexte pirandellien.

6 C'est sur ce dernier point que je souhaite m'attarder, car cette relation d'intertextualité, loin d'être la plus flagrante à l'échelle du roman puisqu'elle n'apparait clairement qu'en deux occasions (pp. 14 et 74), n'en contribue pas moins de façon décisive à préciser la théorie du personnage et, plus généralement, de la littérature qui sous-tend le roman.

\section{« HUBERT}

Voici donc. Je me présente: Hubert Lubert, romancier de profession, de vocation même et j'ajouterai d'un certain renom. Etant romancier, j'écris donc des romans. Ecrivant des romans, j'ai affaire à des personnages. Or voici que l'un d'eux vient de s'éclipser. Textuellement. Un roman que je venais de commencer, une dizaine de pages environ, quinze au plus, et dans lequel je mettais les plus grands espoirs, et voilà que le personnage principal, à peine esquissé, disparait. Comme je ne puis évidemment continuer sans lui, je viens vous demander de le retrouver.

MORCOL (rêveusement)

Voilà qui est bien pirandellien.

HUBERT

Pirandellien?

MORCOL

Un adjectif dérivé de Pirandello. C'est vrai, vous ne pouvez comprendre.

HUBERT

Un client?

MORCOL

Chut ! Revenons au fait. Comment se présentait votre bonhomme?» (p. 14)

7 Cette indication explicite d'un intertexte pirandellien par l'insistance sur son anachronisme compte tenu de l'ancrage temporel de l'histoire racontée (fin du 19 ème siècle) sera ultérieurement confirmée et précisée à la faveur d'une syllepse intertextuelle :

HUBERT (devant une feuille de papier blanc)

[...] Peut-être un jour en sera-t-il ainsi pour tous. Nous n'aurons plus de personnages. Nous deviendrons des auteurs en quête de personnages*. (p. 74)

Ces références ponctuelles constituent bien plus que les occasions d'un simple dévoilement de l'intertexte pirandellien, incontestablement à l'œuvre dans Le Vol d'Icare. Ainsi de la réflexion de Morcol (p.14) qui, par la création d'un effet d'anachronisme, introduit déjà un dysfonctionnement dans le système des personnages, encore à l'état d'ébauche. En effet Morcol, pourtant simple personnage intradiégétique en apparence, démontre ici un savoir paradoxal, presque impossible: la connaissance de l'œuvre 
pirandellienne (le texte italien de Six personnages en quête d'auteur ${ }^{10}$ a été publié en 1958), condition nécessaire de sa réflexion, est incompatible avec son ancrage historique de personnage fin de siècle. A la faveur de cette intervention, Morcol tend à s'extraire de la diégèse pour se rapprocher de l'auteur supposé du Vol d'Icare dont il semble partager l'omniscience ; comme l'indique par deux fois l'affirmation arbitraire de la supériorité de son savoir sur celui des personnages diégétiques : tout d'abord simple constat (« C'est vrai vous ne pouvez comprendre ») puis déni d'information (" Chut ! Revenons au fait »).

Ainsi la désignation de l'intertexte, par-delà son rôle citationnel, remplit-elle un office plus complexe, et contestataire : en introduisant un dysfonctionnement dans le système des personnages, elle permet d'attirer l'attention des lecteurs sur l'arbitraire qui préside à son élaboration - ce qui revient à en souligner la fictivité. Par le recours à ces manipulations métaleptiques, le scripteur empêche le fonctionnement de l'illusion référentielle, la mise en place de l'effet de réel ; déjà contrecarrés par les nombreuses séquences métatextuelles. Les lecteurs, confrontés à ces dysfonctionnements, ces déca lages, ces glissements, ne peuvent plus voir dans le roman qu'ils sont en train de lire une banale tentative pour représenter le réel, mais sont conduits bon gré mal gré à esquisser une hiérarchisation des personnages, à s'interroger sur les concepts de personnage et de personne, à se voir lire un roman en train de se déconstruire au rythme auquel s'évadent les personnages...et dans le même temps de se construire - comme l'indique l'excipit :

HUBERT (refermant son manuscrit sur Icare)

Tout se passa comme prévu ; mon roman est terminé. (p. 253).

D'autre part, il convient d'apprécier la pertinence du rapprochement effectué par Morcol : une révélation à la faveur de laquelle, en même temps que s'effectue un dépla cement d'un univers intradiégétique à un univers extradiégétique, la chronologie s'inverse. Pirandello devient un auteur non plus postérieur à Morcol, et donc nécessairement inconnu de lui, mais antérieur de dix ans à Raymond Queneau écrivant Le Vol d'Icare. Voici peut-être divulgué par Morcol le «secret de fabrication» du roman de Queneau: un livre (Six personnages en quête d'auteur) à l'origine d'un autre livre (Le Vol d'Icare). La pièce de Pirandello peut être perçue comme argument, point de départ du roman de Queneau, qui consisterait donc en l'appropriation d'une topique, ultérieurement renversée, décalée et développée : le vol de Queneau, larcin signalé dans un geste métatextuel au début du roman dont il constitue la condition de possibilité.

11 Mais l'appropriation est à tel point originale que par un nouveau renversement Le Vol d'Icare devient origine des Six personnages en quête d'auteur - le roman de Queneau constituant non pas une simple citation mais une «traduction » de la pièce de Pirandello, car la prise de la parole de l'autre aboutit à la restitution d'une parole autre. Comme l'indique la permutation des substantifs symétriques contenus dans le titre de la pièce (les "personnages en quête d'auteur » devenant des «auteurs en quête de personnages " - p. 74), Queneau pratique un renversement total de la perspective pirandellienne, tant en ce qui concerne l'intrigue que la conception de la littérature que son traitement présuppose.

12 En effet, l'argument de son roman semble avoir été suggéré à Queneau non pas tant par la pièce elle-même que par la préface ${ }^{11}$ autographe de Pirandello, qui en raconte et analyse la genèse :

Mais on ne donne pas en vain la vie à un personnage.

Créatures de mon esprit, ces six personnages vivaient déjà d'une vie qui leur était propre et qui n'était plus la mienne, d'une vie qu'il n'était plus en mon pouvoir de leur refuser. 
C'est si vrai que, comme je persistais dans ma volonté de les chasser de mon esprit, eux, déjà entièrement détachés de tout support narratif, personnages d'un roman sortis par miracle des pages du livre qui les contenait, ils continuaient de vivre pour leur propre compte $[. .$.$] .$ cette autonomie « miraculeusement » acquise, fuyant son auteur -, au lieu que Pirandello, lui, a « dépassé » ce stade initial et :

[...] voulu représenter six personnages qui sont à la recherche d'un auteur. Leur drame ne parvient pas à être représenté, précisément parce que fait défaut l'auteur qu'ils cherchent; et ce qui, par contre, est représenté, c'est la comédie de cette vaine tentative qu'est la leur, avec tout ce qu'elle a de tragique du fait que ces six personnages ont été refusés. (p. 18) diversité des media utilisés : d'une part l'écriture, d'autre part les ressources combinées de l'écriture et de la scène. Cette différence essentielle permet en partie d'expliquer les divergences des conceptions du personnage et, partant, de la littérature, propres à Pirandello et Queneau - entreprise qui évidemment nous éloigne des textes, car elle repose sur une activité lectorale d'induction puis de glose ; mais qui me semble inhérente à la réception des mécanismes intertextuels.

Et sur ce point l'intertextualité est complétée par la dimension intensément métatextuelle du roman: ces nombreuses séquences posent en effet des problèmes théoriques capitaux, sur un mode déceptif. Nulle part l'essence purement fictive des personnages ou l'irréductible hétérogénéité du réel et de la littérature ne sont explicitement proclamées avec l'autorité d'une parole que sa source rendrait incontestable: toute prise de position théorique, assumée par un discours qui trouve inévitablement sa réfutation sur le champ de la scène énonciative, est, sitôt énoncée, relativisée ; le sens déçu.

Toutefois, même s'il s'agit sans doute de l'attitude la plus sage, la plupart des lecteurs ne sauraient se contenter indéfiniment de cette suspension du jugement critique qui leur est de la sorte imposée ; d'autant qu'en dépit de cette déception du sens théorique la pratique de composition et d'écriture à l'œuvre dans le roman actualise un certain nombre de choix narratifs, énonciatifs, stylistiques et génériques lourds de présupposés. C'est donc le travail de textualisation même qui est incitation des lecteurs à un effort d'induction, la perpétuelle déception du sens pouvant être lue comme un encouragement à saturer l'ellipse ainsi créée, par l'émission d'hypothèses relatives à la conception de la littérature sous-jacente à la production du texte.

Ainsi les traitements convergents de l'intertexte et du métatexte et leur influence sur le système des personnages dans Le Vol d'Icare me semblent-ils révélateurs d'une conception moderniste de la littérature, qui conteste certains des principes de base du récit réaliste «traditionnel«, comme l'effet-personnage et son corollaire, l'illusion référentielle. Au contraire, ces principes, symptomatiques d'une conception « idéaliste, anthropocentrique et personnaliste $»^{12}$ de l'œuvre littéraire, informent clairement le discours préfaciel pirandellien :

Ils se sont déjà détachés de moi ; ils vivent pour leur propre compte ; ils ont acquis voix et mouvement; ils sont devenus d'eux-mêmes, dans cette lutte pour la vie qu'ils ont dû livrer contre moi, des personnages qui peuvent bouger et parler tout seuls; ils se voient déjà comme tels ; ils ont appris à se défendre de moi ; ils sauront aussi se défendre des autres. (Pirandello, op. cit., p. 15) 
dépit du postulat commun aux deux œuvres (la possibilité d'une vie autonome des personnages), on comprend désormais comment il est possible de lire chez Queneau un total renversement de la perspective pirandellienne, non seulement anecdotique mais plus encore théorique. Les déclarations de la préface l'attestent: malgré certaines "fulgurances » (coprésence de personnages appartenant à divers plans de réalité, naissance sur scène du personnage de Madame Pace), Pirandello souscrit encore largement à une conception personnaliste, anthropocentrique et idéaliste de la littérature, ce qui peut partiellement s'expliquer par son statut d'homme de théâtre. Les personnages de théâtre, contrairement aux personnages de roman, ne peuvent être entièrement considérés comme de pures fictions littéraires dans la mesure où ils ne sont réellement achevés qu'à partir du moment où des acteurs les incarnent sur scène. C'est d'ailleurs la possibilité paradoxale d'un drame de l'inachèvement qui est au cœur de la représentation dans Six personnages en quête d'auteur. lecteurs à l'effet-personnage, horizon d'attente typique d'un état historiquement déterminé de la conception de la littérature. Or Le Vol d'Icare conteste cette attitude lectorale en la rendant textuellement, c'est-à-dire dans et par le texte, impossible. Conformément au vocabulaire de la critique prémoderniste, les personnages y sont dépouillés de toute profondeur, de toute épaisseur, de toute densité psychologique et se réduisent a contrario à la seule consistance du nom propre ou commun qui les désigne lieu où se rencontrent, parfois s'affrontent, parfois se confondent fonction et fiction ${ }^{13}$.

Si l'attention portée à la relation d'intertextualité qui unit le roman de Queneau à la pièce de Pirandello peut permettre aux lecteurs de spéculer par induction sur les convictions théoriques respectives de ces deux auteurs, l'examen des spécificités textuelles du Vol d'Icare vient amplement confirmer ces hypothèses. S'y joue en effet un refus permanent des habituels procédés vraisemblabilisants : - par l'insistance, lors des nombreux passages métatextuels, sur le statut fictif (intravoire métadiégétique) de certains personnages. - par la multiplication ostentatoire et ludique des phénomènes de métalepse narrative. Considérés en régime réaliste comme autant de dysfonctionnements, ils valent ici indication de fictivité voire, compte tenu de leur fréquence, d'hyperfictivité. - par une dialectique quelque peu perverse entre les concepts d'autonomie du personnage et d'arbitraire narratif, qui joue sur l'entière diachronie du récit et ne se résout expli citement qu'à la toute dernière ligne du roman. Si le postulat personnaliste parait tout d'abord retenu puisque les personnages semblent vivre pour leur propre compte, il n'en est que plus brutalement récusé in fine. Le Vol d'Icare opère dans sa ph(r)ase ultime (coup de...théâtre ?) un total renversement du sens. La coprésence des personnages sur divers plans de réalité ainsi que leurs escapades métaleptiques constituaient certes déjà autant d'indications implicites de leur fictivité, mais la lettre du texte proclamait leur autonomie et leur réalité : l'excipit, révélant l'existence d'un plan, affirme la servitude d'Icare, son statut fictif, ainsi que l'omnipotence du scripteur.

Cette remise en cause tardive du postulat-poncif initial me semble revêtir une valeur de leçon, et s'inscrire en conclusion de la graduelle et ironique propédeutique à la théorie du personnage qu'est aussi ce roman. Il s'agirait d'une mise en garde ludique contre une fréquente " erreur » de lecture, ainsi corrigée. Sur ce point, le roman de Queneau peut paraître anticiper la réflexion théoricienne de Philippe Hamon : 
[La vie des personnages] est [...] toujours interprétée en termes de vérité, de représentation, d'autonomie, de réalisme psychologique fondé sur une expérience, jamais en termes de cohérence romanesque, de logique narrative, jamais comme l'expérimentation ou la manifestation de règles logiques et stylistiques contraignantes. Or des études plus fines montrent aisément que « l'effet de vie » est d'abord lié à la mise en œuvre de techniques rhétoriques et stylistiques précises [...] ${ }^{14}$.

La pratique d'écriture actualisée dans Le Vol d'Icare permet donc dans une large mesure d'induire la conception que pouvait se faire Queneau de la littérature dans son rapport à la réalité. La rupture avec les théories idéalistes, psychologistes et personnalistes, liées à la valorisation d'une esthétique de la représentation et de la conception unitaire classique de la personne, indique clairement qu'il n'est pas question pour lui de confondre littérature et réalité. Même le paradoxe qui semble frapper d'incertitude la frontière de la représentation («Ils [les autres gens dans les rues] sont peut-être les personnages d'une autre espèce d'auteurs. » (p. 215) $\left.{ }^{15}\right)$ ne peut apparaître et se développer qu'en vertu des pouvoirs du texte, en l'occurrence à la faveur des métalepses narratives.

La pratique d'écriture quénienne actualise une conception hyperlucide de la littérature, conçue comme travail le plus souvent ludique sur les signifiants, « expérimentation ou manifestation de règles logiques et stylistiques contraignantes et spécifiques " (Hamon, op. cit., p. 13). Que l'on songe notamment dans Le Vol d'Icare au rôle joué par l'intertexte - ou plutôt les intertextes: outre l'ancrage historique fin de siècle, occasion d'une parodie, le roman peut en effet être perçu comme le produit de la colli/usion de deux intertextes majeurs: la pièce de Pirandello / "la mythologie ancienne» (p. 189), plus précisément le mythe d'Icare. "Empruntée » à Pirandello, l'autonomie (ici seulement apparente) du personnage n'est que la condition de possibilité du cheminement diachronique d'Icare vers le destin, c'est-à-dire le dénouement narratif, qu'imparablement lui assigne le déterminisme de son nom propre.

A cette occasion, on mesure à quel point l'intertextualité est susceptible de fonctionner comme facteur de dynamisme générique: la pièce de théâtre et le récit mythologique sont relus (traduits) et confrontés au sein d'un roman, qui paradoxalement devient leur commune origine, et opère en conséquence une transgression des genres canoniques, codifiés. Roman (en raison de l'abondance de personnages, du développement et de la résolution d'une intrigue; et surtout du maintien de l'intitulé à valeur de définition générique dans le paratexte) ou pièce de théâtre imprimée (pour l'organisation de la scène énonciative, les modalités de circulation de la parole)? Le Vol d'Icare témoigne ironiquement d'une forte tension entre ces deux modes littéraires institués, et contribue ainsi à rendre caducs des cloisonnements génériques sans doute trop stricts et figés, en manifestant la résistance des textes singuliers à l'abstraction, voire leur irréductibilité à un modèle matriciel qui prétendrait les transcender.

Cette contestation tout à fait consciente, puisque dialectisée au cours d'une séquence métatextuelle (p.112), des genres institués trouve un écho dans l'opposition de Queneau (déductible du traitement particulier du système des personnages dans Le Vol d'Icare) à l'idéologie personnaliste. Il s'inscrit ainsi dans un «mouvement » de réaction contre les excès de sacralisation du personnage comme personne psychologique vivante, venu d'horizons divers: la sociologie et l'anthropologie, la psychanalyse freudienne, les structuralismes, les diverses linguistiques ${ }^{16}$, et bien sûr les romanciers eux-mêmes, qui ont développé durant les années 50 une critique radicale des présupposés idéologiques de 
l'esthétique de la représentation et des approches traditionnelles du concept de personnage.

Toutefois, la contestation menée par Queneau se distingue très nettement de celle des diverses avant-gardes romanesques : à la lecture du Vol d'Icare, si le confusionnisme naïf entre personnage et personne devient proprement impossible, la première de ces deux notions n'en demeure pas moins l'élément central, focal du récit - ce qui constitue un indéniable facteur de lisibilité. La conception «traditionnelle » du personnage est certes contestée, mais jamais au prix d'une disparition totale ou d'une fragmentation radicale. $\mathrm{Au}$ contraire, la subversion du concept s'effectue par le biais d'une sur-valorisation, hyperbolique, caricaturale et ironique. Une ironie d'ailleurs dédoublée, qui vise non seulement les romanciers réalistes, mais aussi certains "Nouveaux Romanciers", convaincus de l'impossibilité d'écrire le destin des personnages. C'est de ce choix paradoxal que provient l'originalité de ce roman qui, pour lutter contre les prestiges de l'illusion référentielle, joue du point qui constitue le plus important facteur d'« erreurs » de lecture : fascination, identification, projections.

Si le lien entre intertextualité et théorie est aussi spectaculaire dans Le Vol d'Icare, c'est bien sûr pour partie en raison de l'esthétique moderniste dont participe ce roman ; mais cette corrélation informe à quelque degré toute production littéraire intégrant des opérations intertextuelles, ce qui peut s'expliquer par les liens indissolubles de l'intertexte et du métatexte. Comme je l'affirmais en préambule, la relation d'interlocution de texte à texte(s) implique toujours, plus ou moins, une relation de commentaire. Ainsi, les pratiques du plagiat (évidemment), mais aussi de la citation explicite ou implicite et de l'allusion manifestent un écart variable qui prend valeur de glose. Citer X ou Y, explicitement ou implicitement, équivaut à adopter volontairement ou non à son égard une position spécifique sur les plans historique, idéologique, esthétique et théorique ; et à son tour, comme le signale Stefan Morawski, cette position recouvre un certain nombre de fonctions : «fonction d'érudition, invocation d'autorité, fonction d'amplification, fonction ornementale $»{ }^{17}$

31 L'intertextualité manifeste donc à la fois, de façon quelque peu contradictoire, une relation d'appartenance et un écart. Mais cette apparente contradiction n'est guère surprenante puisqu'elle provient de l'essence même du texte littéraire : un système dont les divers éléments constitutifs jouent les uns par rapport aux autres (ce qui inclut les rapports de coréférence), mais qui est toujours susceptible de se voir confronter à d'autres systèmes singuliers. En d'autres termes, si les textes littéraires parlent du monde, ils parlent aussi d'eux-mêmes et d'autres textes; et l'intertextualité pourrait également être définie comme l'ensemble des «frictions $~^{18}$ qui apparaissent au point de contact de ces différents « textes-systèmes ». Si la théorie lui est consubstantielle, c'est en raison du positionnement frontalier que requiert son émergence, et qui implique une dialectique du même (la littérature) et de l'autre (chaque texte dans sa singularité), de la clôture et de son effraction ${ }^{19}$. Voilà qui, assurément, vaut bien une heure de peine ${ }^{20}$. 


\section{NOTES}

1. Julia Kristeva, Sèméiotikè, Paris, Seuil, 1969.

2. Antoine Compagnon, La Seconde main (Ou le travail de la citation), Paris, Seuil, 1979.

3. Michael Riffaterre, Essais de stylistique structurale, Paris, Flammarion, 1971 pour la traduction française, collection " Nouvelle bibliothèque scientifique ». La Production du texte, Paris, Seuil, 1979 pour la traduction française, collection « Poétique ». Sémiotique de la poésie, Paris, Seuil, 1983 pour la traduction française, collection « Poétique ».

4. Gérard Genette, Palimpsestes (La littérature au second degré), Paris, Seuil, 1982, collection «Poétique », p. 8.

5. Ibidem.

6. C'est par exemple l'opinion de Bernard Magne : « La relation d'appartenance fonde la synecdoque métatextuelle, dont la forme la plus stricte est la citation implicite ou, comme je l'ai risqué ailleurs, implicitation. » (" Métatextuel et lisibilité », Québec, Protée, vol. 14, nº 1-2, printemps-été 1986, p. 83).

7. Ces premières lignes peuvent paraître manifester une forme d'indécision entre analyse de phénomènes concernant l'auteur et son texte et prise en compte des mécanismes de réception. Il ne s'agit pas de quelque maladresse involontaire, mais d'un parti pris méthodologique : reporter l'attention théorico-critique sur l'espace mixte de l'écriturelecture, en vue d'étudier le phénomène littéraire dans sa pluralité constitutive.

8. Raymond Queneau, Le Vol d'Icare, Paris, Gallimard, 1968, collection « blanche ». 9. « Jacques / Je voudrais donner l'impression de la couleur mauve » (p. 52), « Jacques / Mon Chamissac-Piéplu a failli se battre en duel, mais je réserve cet épisode pour plus tard. Après l'adultère qui vient d'être consommé. Dans un fiacre » (p. 101).

10.* C'est moi qui souligne.

Luigi Pirandello, Six personnages en quête d'auteur, 1958 pour le texte italien ; Paris, Gallimard, 1977 pour la traduction française, rééditée dans la collection « Folio ». 11. Pirandello, ibidem, p. 14.

12. Philippe Hamon Le Personnel du roman : le système des personnages dans les RougonMacquart d'Emile Zola, Genève, Droz, 1983. Cet ouvrage constitue ma principale référence pour toutes les analyses du traitement des personnages dans Le Vol d'Icare.

13. Les spécificités onomastiques, qui contribuent à régir l'itinéraire narratif des personnages dans une relation de simultanéité avec l'intertexte, sont en effet signifiantes. Par exemple, Hubert Lubert est celui qui donne la berlue, qui éberlue : l'illusionniste, le mystificateur qui attend la dernière ligne du roman pour révéler son statut d'auteur. Chez Surget, la fiction détermine la fonction. Nommé, il doit mériter son nom : d'où son duel perdu, et l'intervention chirurgicale (les points de surjet - p. 113) à valeur de baptême. Mandé, valet de Jacques, présente quant à lui un net déficit fictionnel en regard de son poids fonctionnel, qui joue le rôle de déterminisme onomastique : fréquemment mandé (par statut), il devient Mandé. Ce ne sont là que quelques exemples d'un phénomène dont la densité et l'importance à l'échelle du roman exigeraient une analyse autonome.

14. Philippe Hamon, op. cit., p. 13. 
15. Paradoxe analysé par Gérard Genette : « Le plus troublant de la métalepse est bien dans cette hypothèse inacceptable et insistante, que l'extradiégétique est peut-être toujours déjà diégétique, et que le narrateur et ses narrataires, c'est-à-dire vous et moi, appartenons peut-être encore à quelque récit ». (Figures III, Paris, Seuil, 1972, collection «Poétique », p. 245).

16. Cf. Philippe Hamon, pp. 13-18.

17. Stefan Morawski, «The Basic Functions of Quotation », Sign, Language, Culture, Janua Linguarum, Series major, I, La Haye-Paris, Mouton, 1971, pp. 690-705 ; cité par Antoine Compagnon, op. cit., p. 99.

18. Cf. Antoine Compagnon, p. 43.

19. A ce propos, je ne pense pas, comme certains (notamment Thomas Pavel, Univers de la fiction, Paris, Seuil, 1988 pour la traduction française, collection « Poétique », p. 150), que l'intertextualité soit incompatible avec la prise en compte de la référentialité. Même si cette théorie a incontestablement eu tendance par le passé à substituer le monde des livres au monde tout court (comme on le voit clairement à la lecture des travaux de Michael Riffaterre), il ne faut pas perdre de vue le fait qu'en elle-même elle provoque une notable effraction de la clôture textuelle (de la clôture de chaque texte particulier sur luimême) et peut donc servir à la remise en cause des hypothèses structurales internalistes, et permettre indirectement de redonner à terme toute son importance à la problématique de la représentation.

20. Au moment de conclure cette réflexion sur l'intertextualité, je n'ai pu résister à la tentation de jouer à mon tour de ces ressources. Chacun aura reconnu la formule sur laquelle s'achève Palimpsestes; et si je n'ai pas fait fausse route au cours de mon raisonnement, tirera de cette appartenance et de cet écart les conclusions théoriques qui lui sembleront s'imposer.

\section{RÉSUMÉS}

La relation d'interlocution de texte à texte(s) que l'on nomme intertextualité est également toujours, à quelque degré, relation de commentaire - à valeur historique, idéologique, esthétique et théorique. Ce lien indéfectible peut en grande partie s'expliquer par la proximité des concepts d'intertexte et de métatexte. Prenant appui sur ce postulat, je m'efforce de démontrer dans cet article que l'intertexte pirandellien à l'œuvre dans Le Vol d'Icare, roman de Raymond Queneau, induit deux conceptions de "l'effet-personnage", et partant de la littérature, radicalement opposées. Plus généralement, je souhaiterais prouver qu'intertextualité et théorie sont consubstantielles, en raison de la dialectique de l'appartenance et de l'écart inhérente aux pratiques allusives et citationnelles. 